\title{
ESPELHOS ESFÉRICOS CONFECCIONADOS COM MATERIAIS ACESSÍVEIS PARA DEMONSTRAÇÃO DE FORMAÇÃO DE IMGENS EM SALA DE AULA ${ }^{+*}$
}

\author{
Wellington Luis de Almeida \\ Aluno de graduação \\ Licenciatura em Física \\ Universidade Estadual de Ponta Grossa - UEPG \\ Ponta Grossa - PR \\ Fabiano Meira de Moura Luz \\ Aluno de mestrado \\ Programa de Pós-Graduação em Ciências/Física \\ Universidade Estadual de Ponta Grossa - UEPG \\ Ponta Grossa - PR \\ Jeremias Borges da Silva \\ Silvio Luiz Rutz da Silva \\ André Maurício Brinatti \\ Departamento de Física - DEFIS \\ Universidade Estadual de Ponta Grossa - UEPG \\ Ponta Grossa - PR
}

\section{Resumo}

O conhecimento do assunto espelhos esféricos da óptica geométrica torna-se importante para contribuir com a formação de um indivíduo cujas habilidades e competências promovidas estejam em acordo com o que se espera de um educando em relação ao seu conhecimento de Física em nível de ensino médio. No entanto, para muitos estudantes do ensino médio e fundamental é difícil fazer

\footnotetext{
Spherical mirrors made of available material to demonstrate image formation in classroom

* Recebido: novembro de 2012. Aceito: abril de 2013.
} 
a conexão entre a teoria e a prática, sendo necessárias alternativas compreendidas entre aulas teórico-experimentais com materiais didáticos e métodos que auxiliem alunos a entenderem e admirarem os fenômenos envolvidos nos assuntos enfocados. Assim, este trabalho tem o intuito de corroborar com o educador propondo a confecção de um conjunto didático para o estudo de espelhos esféricos, a saber: espelhos esféricos côncavo e convexo (de vidro de relógio de $50 \mathrm{~mm}$ de diâmetro) com suporte, anteparo e fonte de luz e espelhos esféricos côncavo e convexo (de vidro de relógio de $150 \mathrm{~mm}$ de diâmetro), cujos resultados têm imagens formadas com boa nitidez e torna viável a utilização do conjunto em análises quantitativas, essenciais em situações de ensino-aprendizagem em aulas teórico-experimentais.

Palavras-chave: Conjunto didático. Óptica geométrica. Ensino de Física.

\begin{abstract}
The knowledge of spherical mirrors in Geometrical Optics is highly relevant as it contributes with the instruction of an individual whose abilities and skills are in agreement with what is expected from a High School student regarding Physics at this level. However, for most students in Elementary and High School it is very hard to relate theory and practice, and alternatives involving theoretical-experimental classes with specific teaching material as well as methods that help students to observe and understand Physics phenomena. Thus, this study aims at supporting the educator proposing the manufacturing of a teaching set to study spherical mirrors, that is, concave and convex mirrors (50mm diameter clock glass) with support, backstop and light source; and concave and convex mirrors (150mm clock glass), which present good quality images and make it possible to use the set in quantitative analysis, essential in teaching and learning situations within theoretical-experimental lessons.
\end{abstract}

Keywords: Teaching set. Geometrical Optics. Physics teaching. 


\section{Introdução}

O estudo da óptica geométrica foi responsável por vários avanços na ciência. A construção de lupas, lunetas, telescópios, microscópios, sistemas de transmissão de dados com alta velocidade, como a fibra óptica, entre outros, só foi possível devido ao estudo da óptica geométrica, e a compreensão dos fenômenos nela envolvidos.

Ao levar em consideração que a compreensão da ciência e da tecnologia deve promover habilidades e competências no educando de forma a contribuir com sua autonomia (HOSOUME; KAWAMURA; MENEZES, 1998; MENEZES, 2000; KAWAMURA; HOSOUME, 2003), acredita-se que ter o conhecimento deste tema pode promover habilidades e competências em acordo com o que se espera de um educando em relação à Física em nível de ensino médio (BRASIL, 2002; KAWAMURA; HOSOUME, 2003; BRASIL, 2006).

Sabe-se também que para muitos estudantes do ensino médio e fundamental é difícil fazer a conexão entre a teoria e a prática em aulas de física, principalmente porque nas aulas normalmente não há descoberta ou redescoberta. A contextualização é dificultada pela falta de material adequado para uma prática ilustrativa e compreensiva. Então, são necessárias alternativas compreendidas entre aulas teórico-experimentais com materiais didáticos e métodos que auxiliem alunos a entenderem e admirarem os fenômenos envolvidos nos assuntos abordados.

Há vários trabalhos na literatura que abordam o tema óptica geométrica ou assuntos pertinentes a esse com vários enfoques para o ensino em nível médio e ou superior. Especificamente, em relação a lentes e espelhos esféricos, nos trabalhos de Pimentel e Brinatti (1989) e de Silva (2004) têm-se propostas de confecção de banco óptico para estudo e compreensão de lentes esféricas. Nos trabalhos de Pimentel (1989) e Figueredo e Bertuola (2007) há propostas de confecção de material didático para auxiliar nos traçados de raios notáveis na construção de imagens. Misoguti et al. (1997), com o uso de materiais de laboratório e algumas adaptações, trazem uma demonstração da importância da refração da luz no funcionamento de lentes esféricas. Catelli e Reis (2004) e Catelli e Vicenzi (2004) relatam experiências de aprendizagem sobre a reflexão da luz em espelhos esféricos em uma situação fora da sala de aula e em outra originada em sala de aula, respectivamente. Silveira, Axt e Pires (2004) tratam, de forma detalhada, a formação de imagens em espelhos côncavos incluindo a esse sistema óptico a lente de nosso olho e Costa e Almeida (2005) trazem uma discussão de formação de imagens de espelhos e lentes tridimensionais, essas raramente abordadas em sala de aula. 
Assim, com o intuito de corroborar com o educador em situações de ensino-aprendizagem, tais como demonstrações em sala de aula, proposição de montagem de material pelos alunos para experimentos em aula ou em feiras de ciências, sobre o assunto espelhos esféricos da óptica geométrica que se insere no tema: Som, Imagem e Informação dos Parâmetros Curriculares Nacionais para o Ensino Médio (BRASIL, 2002) e das Orientações Curriculares para o Ensino Médio (BRASIL, 2006), fornece-se, no presente trabalho, uma opção de material para o uso no processo de ensino-aprendizagem desse assunto.

\section{Fundamentação teórica}

A óptica geométrica tem grande importância na história da ciência e da tecnologia como foi mencionado anteriormente. Este ramo da óptica possibilita usar simplificações, como raios luminosos e sua propagação retilínea, para explicar fenômenos de reflexão e refração da luz, desde que o comprimento de onda da onda eletromagnética em questão, neste caso luz visível, seja muito pequeno se comparado com o tamanho do objeto, ou sistema físico, que a mesma atravessa (FEYNMAN; LEIGHTON; SANDS, 1964; TIPLER; MOSCA, 2009). Para o presente estudo, usou-se destas simplificações e, portanto, da óptica geométrica.

Considere os dois espelhos esféricos representados na Fig. 1 e seus elementos, a saber: eixo principal do espelho, a reta que contém $\mathrm{V}$ e $\mathrm{C}$; vértice do espelho, V; centro de curvatura do espelho, $\mathrm{C}$; ponto focal do espelho, F; raio de curvatura do espelho, $R$. De acordo com a convenção adotada, tem-se: $R>0$ se $\mathrm{C}$, está do mesmo lado da superfície que a luz refletida, situação para o espelho côncavo (Fig. 1a), caso contrário, $R<0$, situação para um espelho convexo (Fig. 1b).

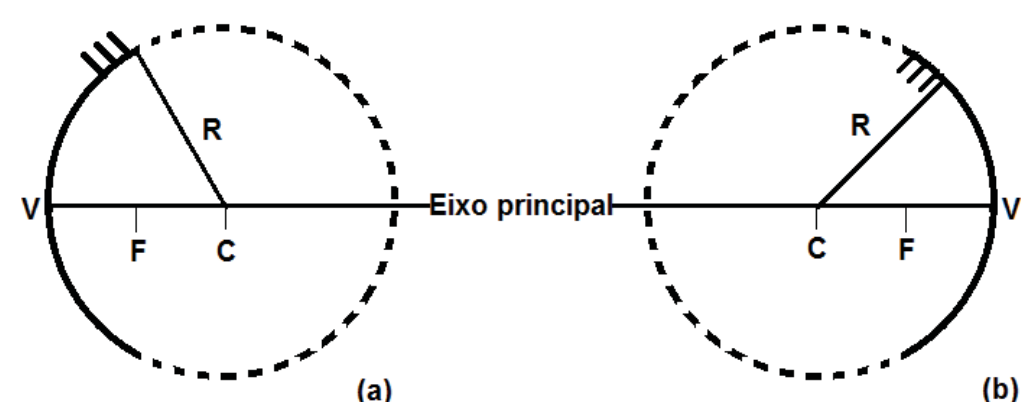

Fig. 1 - Representações dos elementos de espelhos esféricos. a) côncavo; b) convexo. 
Para um espelho esférico côncavo (Fig. 1a.), sabe-se que a distância focal, $f$, distância entre $\mathrm{V}$ e $\mathrm{F}$ ou entre $\mathrm{F}$ e $\mathrm{C}$, pode ser determinada a partir de $R$, dada pela equação (1),

$$
f=\frac{1}{2} R
$$

Ou utilizando a equação do espelho, dada pela equação (2), onde $p$ é a distância do objeto ao espelho e $p^{\prime}$ e a distância da imagem ao espelho,

$$
\frac{1}{f}=\frac{1}{p}+\frac{1}{p^{\prime}}
$$

E, por convenção, se adota: $p>0$, o objeto está no mesmo lado da superfície que a luz incidente e o objeto é real, caso contrário, $p<0$, o objeto está no lado oposto da superfície que a luz incidente e o objeto é virtual; $p^{\prime}>0$ a imagem está no mesmo lado da superfície que a luz refletida e a imagem é real, caso contrário, $p^{\prime}<0$, a imagem está no lado oposto da superfície que a luz refletida e a imagem é virtual.

A formação de imagens em espelhos esféricos para objetos extensos é determinada utilizando os raios notáveis, ou raios principais, considerando o objeto perpendicular ao eixo de referência do espelho, ou eixo principal, cujos raios principais têm como ponto de partida o ponto limitante da máxima extensão do objeto: a) raio paralelo, este incide paralelamente ao eixo principal e sendo refletido através de F; b) raio focal, este incide na direção que contém $F$, sendo refletido paralelamente ao eixo principal; c) raio radial, este incide na direção que contém $\mathrm{C}$, sendo refletido sobre a mesma direção e coincidindo com a mesma reta e o raio que incide sobre o vértice $\mathrm{V}$ do espelho é refletido simetricamente em relação ao eixo principal. O ponto limitante da máxima extensão da imagem é o ponto onde os raios notáveis se cruzam (FEYNMAN; LEIGHTON; SANDS, 1964; TIPLER; MOSCA, 2009). As várias situações de formação de imagens estão representadas na Fig. 2. Para espelhos esféricos côncavos as situações são representadas nas Figs. 2a a 2e, e para espelhos convexos na Fig. 2f. (FEYNMAN; LEIGHTON; SANDS, 1964; TIPLER; MOSCA, 2009). 


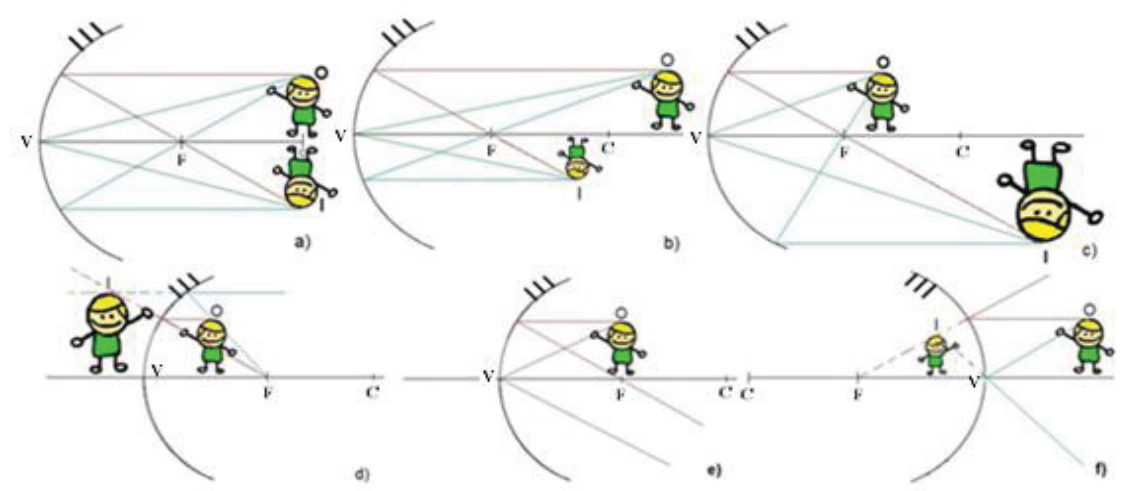

Fig. 2 - Representações das imagens formadas por espelhos côncavos e convexos com o uso dos raios notáveis. O bonequinho com o indice $O$ é objeto em $p$ e sua imagem com indice I em $p^{\prime}$. a) imagem formada por um espelho côncavo quando o objeto está em $C$; b) imagem formada por um espelho côncavo quando o objeto está além $C$; c) imagem formada por um espelho côncavo quando o objeto está entre $C$ e $F$; d) imagem formada por um espelho côncavo quando o objeto está entre $\mathrm{F}$ e $\mathrm{V}$; e) imagem formada por um espelho côncavo quando o objeto esta sobre $F$ (a imagem é formada no infinito), f) imagem formada por um espelho convexo. Nas situações d) ef) as imagens são virtuais.

\section{Objetivo}

Confecção de um conjunto didático para o estudo de espelhos esféricos contendo os seguintes dispositivos: espelhos esféricos côncavo e convexo de vidro de relógio de $50 \mathrm{~mm}$ de diâmetro com suporte, anteparo, fonte de luz. Fica como opção a confecção dos espelhos esféricos côncavo e convexo de vidro de relógio de $150 \mathrm{~mm}$ de diâmetro.

\section{Materiais e métodos}

Primeiramente, descrevem-se os materiais e procedimentos utilizados para a confecção do conjunto didático para o estudo dos espelhos esféricos e, em seguida, os métodos de determinação da distância focal dos espelhos confeccionados. É necessário ressaltar que os dispositivos confeccionados têm como ponto de partida o trabalho de Pimentel e Brinatti (1989).

Para a confecção dos espelhos foram utilizados: 
$\checkmark 8$ vidros de relógio de $50 \mathrm{~mm}$ de diâmetro;

$\checkmark 2$ vidros de relógio de relógio de $150 \mathrm{~mm}$ de diâmetro (opcional);

$\checkmark 1$ rolo de fita cromo;

$\checkmark 1$ rolo de fita adesiva;

$\checkmark$ cola PVC;

$\checkmark 80 \mathrm{~cm}$ (aproximadamente) de cano de PVC com diâmetro de $50 \mathrm{~mm}$;

$\checkmark 4$ lâmpadas fluorescente de $7 \mathrm{~W}$;

$\checkmark 4$ soquetes;

$\checkmark 4$ interruptores tipo pera;

$\checkmark 4$ plugues de tomada;

$\checkmark 4 \mathrm{~m}$ de fio;

$\checkmark 1,5 \mathrm{~m}^{2}$ de papelão;

$\checkmark 4$ folhas brancas A4;

$\checkmark$ caneta hidrográfica preta;

$\checkmark$ serra de arco;

$\checkmark$ lixas;

$\checkmark$ tesoura;

$\checkmark$ chave de fenda;

$\checkmark$ alicate.

As quantidades acima são para a confecção de quatro conjuntos. Descreve-se a confecção dos espelhos de vidro de relógio de $50 \mathrm{~mm}$, uma vez que os espelhos com vidros de relógio de $150 \mathrm{~mm}$ são apresentados como opção e sua confecção e determinação da distância focal são semelhantes aos de diâmetro menor.

O preço unitário dos vidros de relógio: $50 \mathrm{~mm}(\mathrm{R} \$ 2,00)$ e $150 \mathrm{~mm}(\mathrm{R} \$$ $8,50)$ e a fita cromo, rolo com $50 \mathrm{~m}(\mathrm{R} \$ 14,00)$.

Para confeccionar os espelhos esféricos, utilizaram-se os vidros de relógio e fita cromo. A fita cromo foi cortada, com o auxílio de uma tesoura, na metade de sua largura e essas tiras foram fixadas nas superfícies côncavas e convexas dos vidros de relógio de diâmetro de $50 \mathrm{~mm}$. Os suportes para fixar os espelhos foram confeccionados a partir de anéis de cano de PVC de $50 \mathrm{~mm}$ de diâmetro, cortados com o auxílio da serra de arco e as rebarbas foram aparados com a lixa. Eles foram montados com um anel superior de 1,0 cm de largura sobre uma base de metade de um anel de largura de 1,5 cm. Em seguida, cada espelho foi fixado em um destes suportes com fita adesiva.

A fonte de luz foi feita utilizando $15 \mathrm{~cm}$ de cano de PCV de $50 \mathrm{~mm}$, cortado com o auxílio da serra de arco e as rebarbas foram aparadas com a lixa. Co- 
lou-se, em uma das extremidades do cano, um círculo de papelão de $50 \mathrm{~mm}$ com pequenos furos formando a letra "F" no centro e pintado de preto com caneta hidrográfica. Pela outra extremidade inseriu-se o circuito contendo aproximadamente um metro de fio, o interruptor tipo pera, o plugue para tomada e a lâmpada instalada em um soquete, esse circuito foi confeccionado usando o alicate e a chave de fenda.

O anteparo foi feito utilizando um retângulo de papelão de $(15 \times 20) \mathrm{cm}$ o qual foi colocado uma folha branca, com base de $(7 \times 15) \mathrm{cm}$ cortada ao meio e os quadrados formados foram colocados em lados alternados, também confeccionados com o auxílio da tesoura.

Neste trabalho, determinou-se a distância focal dos espelhos de duas formas diferentes.

Na primeira forma, mediram-se os raios de curvatura de oito vidros de relógio de $50 \mathrm{~mm}$ utilizando um esferômetro (LABURÚ; FERREIRA, 1996), e com o auxílio da equação (1) as distâncias focais foram determinadas. Por convenção, adotaram-se esses valores de distância focal como os valores fornecidos pelo fabricante.

Para a determinação da distância focal experimental dos quatro espelhos côncavos de $50 \mathrm{~mm}$ de diâmetro utilizou-se a relação dada pela equação (2) e um paquímetro. Para essa relação, quando a distância entre o objeto e o espelho e a distância entre a imagem e o espelho forem iguais, ou seja, $p^{\prime}=p$, tem-se que $f=p / 2$ (foco será dado pela metade da distância entre o objeto e o espelho), a imagem será real (projetada) invertida e, de mesmo tamanho que o objeto, conforme representado pela Fig. 2a.

\section{Resultados e discussões}

Na Fig. 3, apresentam-se os materiais confeccionados: espelhos esféricos, côncavo e convexo, de vidros de relógio de $50 \mathrm{~mm}$ de diâmetro com suporte, espelhos esféricos côncavo e convexo de vidros de relógio de $150 \mathrm{~mm}$ de diâmetro, anteparo, fonte de luz e suporte. A fita cromo também é apresentada na Fig. 3 para ilustração.

Na Fig. 4, têm-se as situações das imagens obtidas com o espelho côncavo (vidro de relógio de $50 \mathrm{~mm}$ ) quando: determinou-se a distância focal, $f$, do mesmo cuja distância entre o objeto e a imagem são iguais, $p^{\prime}=p$, de mesmo tamanho e $f=p / 2$ (Fig. 4a) sendo essa situação equivalente à Fig. 2a; o objeto está além do centro de curvatura (Fig. 4b); o objeto está entre o centro de curvatura e o foco 
(Fig. 4c). Essas duas últimas situações são equivalentes a Fig. $2 b$ e 2c, respectivamente.

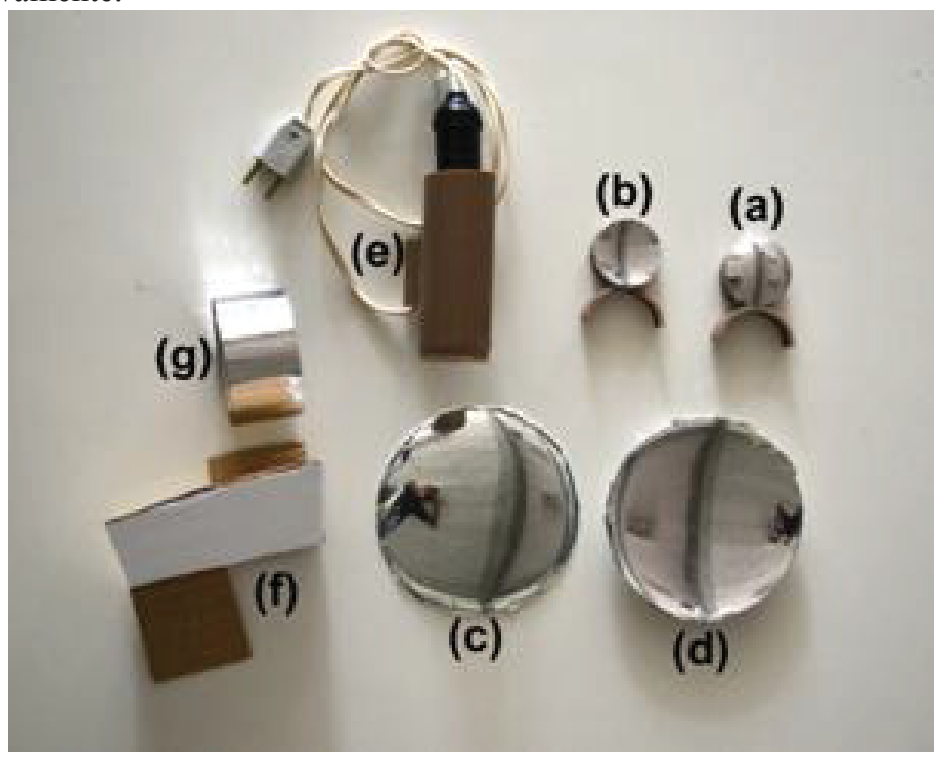

Fig. 3 - a) Espelho convexo (vidro de relógio de diâmetro de $50 \mathrm{~mm}$ ) com suporte; b) espelho côncavo (vidro de relógio de diâmetro de $50 \mathrm{~mm}$ ) com suporte; c) espelho convexo (vidro de relógio de diâmetro de $150 \mathrm{~mm}$ ); d) espelho côncavo (vidro de relógio de diâmetro de $150 \mathrm{~mm}$ ); e) fonte de luz; f) anteparo; g) fita cromo.
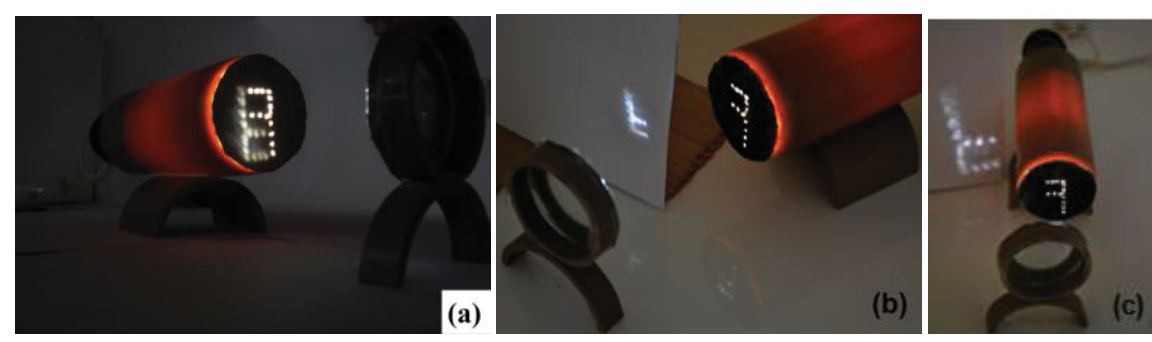

Fig. 4 - Situações das imagens obtidas com o espelho côncavo (vidro de relógio de diâmetro de $50 \mathrm{~mm}$ ): a) objeto no centro de curvatura; b) objeto além do centro de curvatura; c) objeto entre o centro de curvatura e o foco. 
Não foram apresentadas as situações na Fig. 4 relativas às ilustradas na Fig. 2d e Fig. 2e. Na primeira situação, pelo fato do espelho ter uma distância focal pequena que impossibilita a colocação da câmera em uma posição em que fique clara a formação da imagem, e na segunda situação, pelo fato da imagem formar-se no infinito.

Na Fig. 5, tem-se a situação da imagem obtida com o espelho convexo (vidro de relógio de $50 \mathrm{~mm}$ ) que é equivalente a Fig. $2 \mathrm{f}$.

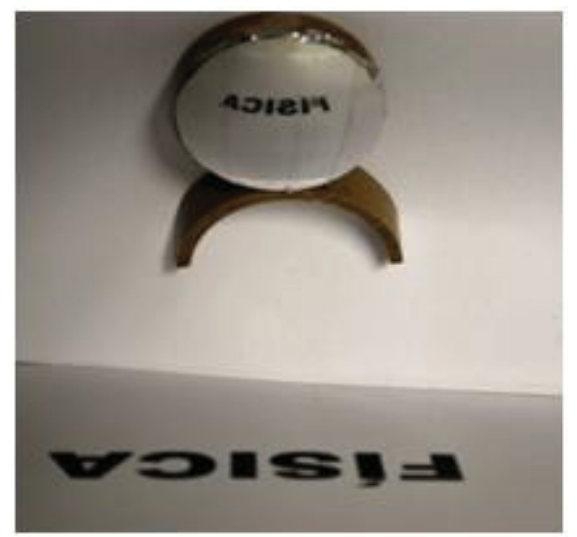

Fig. 5 - Situação da imagem obtida com o espelho convexo (vidro de relógio de diâmetro de $50 \mathrm{~mm}$ ) apresentando o objeto palavra - FÍSICA.

A distância focal dos espelhos esféricos côncavos (vidro de relógio de diâmetro de $50 \mathrm{~mm}$ ), valor do fabricante, por convenção determinada com o esferômetro e equação 1 , foi de $3,64 \pm 0,01 \mathrm{~cm}$, esse praticamente foi o valor medido para os 8 vidros de relógio. A distância focal experimental, por convenção determinada pela equação 2 e uso de um paquímetro, foi de 3,60 $\pm 0,01 \mathrm{~cm}$. Portanto, um erro percentual de $1,09 \%$. Para os espelhos convexos (vidro de relógio de diâmetro de $50 \mathrm{~mm}$ ), adotou-se o mesmo valor de distância focal, em módulo, pois os vidros de relógio apresentaram em média os mesmos raios de curvatura.

Como opção, outros diâmetros de vidro de relógio podem ser escolhidos e confeccionar espelhos de vários raios de curvatura. Na Fig. 6, são apresentados espelhos esféricos com vidro de relógio de $150 \mathrm{~mm}$ de diâmetro, para o caso de espelho esférico côncavo (Fig. 6a) e para o caso de espelho esférico convexo (Fig. $6 b)$. 

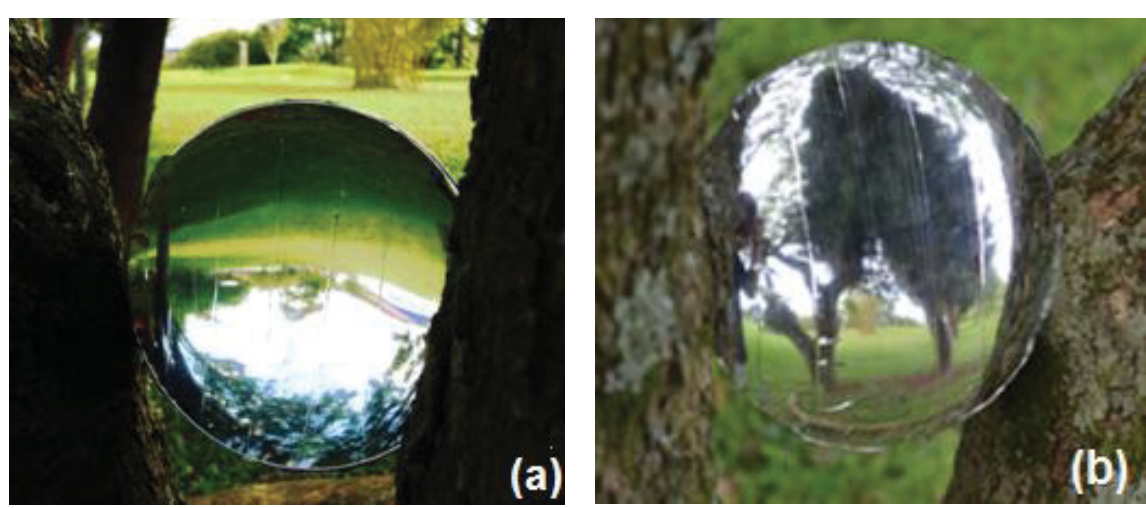

Fig. 6 - Espelhos esféricos confeccionados com vidro de relógio de 150 $\mathrm{mm}$ de diâmetro. a) Imagem formada por um espelho côncavo (objeto além do centro de curvatura); b) Imagem formada por um espelho convexo.

\section{Conclusão}

Como há dificuldades em propor aulas teórico-experimentais em instituições de ensino, principalmente fundamental e médio, devido à carência de materiais didáticos, o material confeccionado apresentado, cujos resultados têm imagens formadas com boa nitidez, torna-se viável em análises quantitativas, essenciais em situações de ensino-aprendizagem em aulas teórico-experimentais em caso de abordagem desse assunto pelos educadores.

\section{Referências}

BRASIL. Ministério da Educação e do Desporto. Secretaria de Educação Média e Tecnológica. PCN+ ensino médio: orientações educacionais complementares aos parâmetros curriculares nacionais: ciências da natureza, matemática e suas tecnologias. Brasília: MEC; SEMT, 2002. 144 p. Disponível em:

$<$ http://portal.mec.gov.br/seb/arquivos/pdf/CienciasNatureza.pdf>. Acesso em: 29 out. 2012.

Secretaria de Educação Básica. Orientações curriculares para o ensino médio: Ciências da natureza, matemática e suas tecnologias. Brasília: Ministério da Educação, Secretaria de Educação Básica, 2006. 135 p. v. 2. Disponível em: $<$ http://portal.mec.gov.br/seb/arquivos/pdf/book_volume_02_internet.pdf $>$. Acesso em: 29 out. 2012. 
CATELli, F., REIS, C. Imagens dentro de lâmpadas. Caderno Brasileiro de Ensino de Física, Florianópolis, v. 21, n. 1, p. 115-119, abr. 2004.

CATELLI, F., VICENZI, S. Óptica geométrica no café da manhã. Caderno Brasileiro de Ensino de Física, Florianópolis, v. 21, n. 3, p. 392-400, dez. 2004.

COSTA, E. V.; ALMEIDA, L. C. Imagens tridimensionais: Formação e análise. Revista Brasileira de Ensino de Física, São Paulo, v. 27, n. 2, p. 231-235, jun. 2005 .

FEYNMAN, R. P.; LEIGHTON, R.B.; SANDS, M. The Feynman Lectures on Physics. Boston: Addison-Wesley Publishing Co., 512 p. v 1. 1964.

FIGUEREDO, M. V.; BERTUOLA, A. C. Bloco projetor manual. Física na Escola, São Paulo, v. 8, n. 2, p. 46-47, out. 2007.

HOSOUME, Y.; KAWAMURA, M. R. D.; MENEZES, L. C. A Física na reforma do Ensino Médio. In: ENCONTRO DE PESQUISADORES EM ENSINO DE FÍSICA, 6, 1998, Florianópolis. Atas... Florianópolis: UFSC, 1998. p. 1-13.

KAWAMURA, M. R. D.; HOSOUME, Y. A Contribuição da Física para o Novo Ensino Médio. Física na Escola, São Paulo, v. 4, n. 2, p. 22-27, out. 2003.

LABURÚ, C. E.; FERREIRA, N. C. Laboratório Caseiro: Construindo um esferômetro e cilindrometro (equipamentos de baixo custo). Caderno Brasileiro de Ensino de Física, Florianópolis, v. 13, n. 2, p. 165-171, ago. 1996.

MENEZES, L. C. Uma Física para o Novo Ensino Médio. Física na Escola, São Paulo, v. 1, n. 1, p. 6-8, out. 2000.

MISOGUTI, L.; MENDONÇA, C. R.; TUBOY, A. M.; HABESCH, R.; BAGNATO, V. S. Lentes esféricas: Uma demonstração para alunos do segundo grau. Revista Brasileira de Ensino de Física, São Paulo, v. 19, n. 4, p. 448-450, dez. 1997.

PIMENTEL, J. R. Painel de traçados de raios. Caderno Catarinense de Ensino de Física, Florianópolis, v. 6, n. 3, p. 248-252, dez. 1989.

PIMENTEL, J. R.; BRINATTI, A. M. Banco ótico e acessórios de baixo custo. Caderno Catarinense de Ensino de Física, Florianópolis, v. 6, n. 1, p. 77-83, abr. 1989.

SILVA, T. R. Banco óptico de baixo custo. Física na Escola, São Paulo, v. 5, n. 1, p. 15 , maio. 2004. 
SILVEIRA, F. L.; AXT, R; PIRES M. A. O que vemos quando nos miramos em um espelho côncavo? Revista Brasileira de Ensino de Física, São Paulo, v. 26, n. 1, p. 19-25, mar. 2004.

TIPLER, A. P.; MOSCA G. Física: para cientistas e engenheiros. Tradução de Naira Maria Balzaretti. 6 ed. Rio de Janeiro: Livros Técnicos e Científicos, 2009. 530 p. v. 2. 